\title{
Reversible Potential Change of Ferrocenylthiol Monolayers Induced by Atomic Force Microscopy
}

\section{AUTHOR(S):}

Ichii, Takashi; Nanjo, Shin-ichiro; Murase, Kuniaki; Sugimura, Hiroyuki

\section{CITATION:}

Ichii, Takashi ...[et al]. Reversible Potential Change of Ferrocenylthiol Monolayers Induced by Atomic Force Microscopy. Japanese Journal of Applied Physics 2009, 48(8): 08JB15.

\section{ISSUE DATE:}

2009-08

URL:

http://hdl.handle.net/2433/123518

\section{RIGHT:}

Copyright (c) 2009 The Japan Society of Applied Physics; This is not the published version. Please cite only the published version.; この論文は 出版社版でありません。引用の際には出版社版をご確認ご利用くださ い。 


\section{Reversible Potential Change of Ferrocenylthiol Monolayers Induced by Atomic Force Microscopy}

Takashi ICHII, Shin-ichiro Nanjo, Kuniaki Murase, and Hiroyuki Sugimura *

Department of Materials Science and Engineering, Kyoto University, Yoshida-honnmachi, Sakyo, Kyoto 606-8501, Japan

Self-assembled monolayers (SAMs) of alkanethiol with a ferrocenyl group (ferrocenylthiol) are well known as electrochemically active ultrathin films. In this study, ferrocenylthiol SAMs were locally modified by applying DC bias voltages to the SAMs using atomic force microscopy (AFM) and the surface potential distributions of the modified areas were investigated by Kelvin probe force microscopy. A positive surface potential shift was detected in the negatively biased regions. In addition, a reversible surface potential change was successfully detected. The surface potential changes were discussed in terms of the electrochemical activity of the ferrocenyl groups.

\section{Introduction}

Alkanethiol self-assembled monolayers (SAMs) have been studied for more than 20 years owing to their precisely controlled thickness and well-ordered structures. They also allow us to easily design and control the surface chemical functionalities of substrates by choosing the terminal functional groups in tethered thiol molecules.

Recently, several research groups have studied ferrocene-terminated alkanethiol (ferrocenylthiol) SAMs as electrochemically active ultrathin films. ${ }^{1-8}$ SAMs containing electrochemically active groups can be employed as an ideal system for studying interfacial electron transfer. The electrochemistry of ferrocene is known to be simple and stable, ${ }^{9}$ and ferrocenylthiols are attempted to be easily formed into uniform monolayers, as shown in Fig. 1. Therefore, they have been expected to be applied to sensors, memory devices and molecular electronics with control of their electrochemical states. Although studies on the macroscopic electrochemical properties of the SAMs are prominently important, microscopic studies of the SAMs are also desired considering the expected applications mentioned above.

Atomic force microscopy (AFM) has been widely used to investigate various surface structures on the nanometer scale. The distribution of material properties of sample surfaces can also be studied using AFM-related techniques. Furthermore, AFM can be used as a nanoscale fabrication tool of sample surfaces. Several fabrication techniques using AFM have been proposed such as atom manipulation, ${ }^{10}$ dip-pen lithography, ${ }^{11}$ and electrochemical modifi-

*E-mail: hiroyuki-sugimura@mtl.kyoto-u.ac.jp 
cation. ${ }^{12,13}$ Electrochemical modification has been widely used because it can be operated in atmospheric condition and it does not require complicated equipment. Most research groups focused on the fabrication of nanoscale oxides on metal or semiconductor surfaces using this technique, so called scanning probe oxidization. Recently, the electrochemical conversions of molecules on substrates using AFM have been reported. ${ }^{14-16}$ Some of them have succeeded in not only oxidizaiton but also reduction of the molecules. In order to construct electronic devices composed of ferrocene derivatives, reversibly manipulating their redox states in the local areas is extremely impressive.

In this study, ferrocenylthiol SAMs were locally modified by applying DC bias voltages to the SAMs using AFM, and the surface potential distributions of the modified areas were investigated by Kelvin probe force microscopy (KFM). Because surface potential of organic monolayers on conducting substrates reflects dipole moment of the molecules, it is expected that the surface potential of the modified area will shift as the redox states of the ferrocenylthiols change.

\section{Experimental Procedure}

11-Ferrocenyl-1-undecanethiol (11-FUT) SAMs and 1-hexadecanethiol (HDT) SAMs on Au substrates were used in this study. 11-FUT and HDT were purchased from Dojindo Laboratories and Aldrich Chemicals, respectively, and used without further purification. Au substrates were prepared by evaporating gold onto Si(100) surfaces (n-type, 1-10 $\Omega \cdot \mathrm{cm}$ ) with a thickness of $150 \mathrm{~nm}$. Before evaporating gold, titanium was evaporated on the Si substrates as an adhesion layer. 11-FUT SAMs and HDT SAMs were prepared by immersing the Au substrates in ethanol solution containing each molecule for $24 \mathrm{~h}$. The concentration and temperature of these solutions were $1 \mathrm{mM}$ and room temperature, respectively. After immersing the samples in the solution, they were rinsed in pure ethanol and dried by $\mathrm{N}_{2}$ flow.

The water contact angles of 11-FUT SAMs and HDT SAMs were typically 82 and $108^{\circ}$, respectively, which indicated that the surface of the samples was covered by ferrocenyl groups for 11-FUT SAMs and methyl groups for HDT SAMs. ${ }^{17}$ We confirmed that no ferrocenyl groups of 11-FUT SAMs in the initial state were oxidized (not in the ferrocenium state), using X-ray photoelectron spectroscopy.

All the AFM and KFM experiments were performed in air or $\mathrm{N}_{2}$ gas using a commercially available AFM instrument (SII NanoTechnology Inc.: HV-300) with home-built equipment for humidity control. Sample surfaces were locally modified by applying DC bias voltages to the sample using contact-mode AFM; the AFM tip is connected to the ground. The KFM experiments were employed with amplitude modulation AFM, where a modulation bias voltage $\left(25 \mathrm{kHz}, 2.0 \mathrm{~V}_{\mathrm{p}-\mathrm{p}}\right)$ was applied to the sample. Highly doped Si cantilevers with Rh coatings (SII NanoTechnology Inc.: SI-DF-3R(100)) were used for both the surface modification and the KFM. The typical spring constant and resonance frequency of the cantilevers were $3 \mathrm{~N} / \mathrm{m}$ 
and $30 \mathrm{kHz}$, respectively.

\section{Results and Discussion}

As schematically illustrated in Fig. 2(a), nine square regions with $1 \times 1 \mu \mathrm{m}^{2}$ of the SAMs were modified by scanning at a rate of $5 \mu \mathrm{m} / \mathrm{s}$ with DC bias voltages from -3.0 to $+2.0 \mathrm{~V}$. Figures 2(b) and 2(c) show a topographic image and the corresponding surface potential image of an 11-FUT SAM after the surface modification operated in air at $50 \%$ relative humidity $(\mathrm{RH})$. The surface potential where DC bias voltages from -3.0 to $-1.5 \mathrm{~V}$ were applied was increased, and no topographic changes were detected in the modified areas. One possible origin of the surface potential shift is the oxidization of the ferrocenyl groups in the 11-FUT SAM. It is simply speculated that the oxidization of ferrocenyl groups will lead to a positive surface potential shift because of the change in their electric dipole moment. Surface potential shift increased as applied negative bias voltage increased, which suggests that the number of oxidized molecules was affected by applied negative bias voltage. In general, surface oxidization using AFM has been performed by applying positive DC bias voltages to the samples while the ferrocenyl groups in the 11-FUT SAMs in this study were oxidized by applying negative DC bias voltages. This can be explained as follows. In the case of the oxidization of metal or semiconductor substrates using AFM, a positive DC bias is applied to the substrate and the substrate is oxidized. On the other hand, in this case, ferrocenyl groups made contacts with an AFM tip with a metal coating, and an insulating layer composed of alkyl chains were inserted between the ferrocenyl groups and the Au substrates. The potential of the AFM tip was relatively high compared with the Au substrates when the negative DC bias voltage was applied to the substrate and the ferrocenyl groups contacting the AFM tip were oxidized.

As a comparative experiment, we also applied the same surface modification on a HDT SAM. Figures 2(d) and 2(e) show a topographic image and the corresponding surface potential image of a HDT SAM after the surface modification. The relative humidity during the modification was also kept at $50 \%$. No surface potential shift was detected in the regions modified with negative DC bias voltages $(-3.0$ to $-0.5 \mathrm{~V})$, which must be because the HDT molecules have no electrochemically active functional groups.

On the other hand, the positively biased $(+1.0,+1.5$, and $+2.0 \mathrm{~V})$ regions of both the samples showed negative surface potential shifts. Because they were found on both the samples, we can conclude that they were not due to the electrochemical change of ferrocenyl groups. One possible reason is the desorption of the molecules. Evans et al. reported that the surface potential of HDT SAMs was higher than that of Au by approximately $600 \mathrm{mV}$, which is due to the electric dipole moment of the adsorbed molecules directing upward from the substrates. ${ }^{18}$ Considering their result, a lower molecular density of HDT molecules will lead to a lower surface potential. Although no surface potential difference between 11-FUT SAMs and Au has been reported to our knowledge, the same discussion may be applicable to the 11-FUT 
SAM. Another possible reason is oxidization of the Au substrate. As we mentioned above, conductive substrates (metals and semiconductors) can be oxidized by applying a positive DC bias. Even if the substrates are covered with organic monolayers, they can still be oxidized. ${ }^{19}$ The surface oxidization will lead to a surface potential shift. In addition, electric charges can be trapped in the oxides. When a positive DC bias is applied to the substrate with oxides, negative charges can be trapped in the oxides, and surface potential will shift negatively. ${ }^{20}$ However, these suggested origins must be accompanied by topographic changes, despite the fact that no topographic changes were detected in this study. Higher-resolution studies should be required to elucidate the origins of the negative surface potential shift.

To examine the effects of adsorbed water and oxygen concentration, the same surface modification was performed on 11-FUT SAMs in air and $\mathrm{N}_{2}$ gas with low humidity. Figures $2(\mathrm{f})$ and $2(\mathrm{~g})$ show a topographic image and the corresponding surface potential image of an 11-FUT SAM modified in air at $10 \%$ RH, respectively, and Figs. 3(h) and 3(i) show a topographic image and the corresponding surface potential image of an 11-FUT SAM modified in $\mathrm{N}_{2}$ at $10 \% \mathrm{RH}$, respectively. Although both samples showed positive surface potential shifts in the regions modified with negative DC bias voltages, the shifts were small compared with that in the 11-FUT SAM modified in air at $50 \%$ RH. Figure $2(\mathrm{j})$ shows the cross-sectional plots obtained along the dotted lines indicated as A-B in Fig. 2(c), C-D in Fig. 2(g), and E-F in Fig. 2(i). Little surface potential shifts were found on the regions modified with smaller negative bias voltages $(-2.0$ and $-1.5 \mathrm{~V})$ in air and $\mathrm{N}_{2}$ gas at $10 \% \mathrm{RH}$; the SAMs modified in air at $50 \% \mathrm{RH}$ showed a few tens of $\mathrm{mV}$ of positive surface potential shift even in the regions modified with a DC bias voltage of $-1.5 \mathrm{~V}$. This result indicates that adsorbed water enhanced the oxidization of the ferrocenyl groups. On the other hand, the positive surface potential shift on the SAM modified with $-3.0 \mathrm{~V}$ in air at $10 \% \mathrm{RH}$ was almost equal to that modified in the $\mathrm{N}_{2}$ gas at the same humidity. That is, no dependence of oxygen concentration on the surface potential shift was found. He et al. reported current measurements between an AFM tip and an 11-FUT SAM using contact-mode AFM in organic solvent and showed a negative differential resistance (NDR), which were discussed from the viewpoint of the electrochemical activity of the SAMs. ${ }^{7}$ They reported that no NDR was found when most oxygen was removed, which did not agree with our study. One possible reason for this difference is the difference in environment. He et al.'s experiment was performed in an organic solvent while ours was performed in air or $\mathrm{N}_{2}$ gas with a certain humidity. There is a possibility that the water meniscus between the tip and the sample in this study contained a small amount of oxygen even under the $\mathrm{N}_{2}$ gas condition and that it was enough to promote the oxidization.

Figures 3(a) and 3(b) show the topographic image and the corresponding surface potential image obtained after the surface modification operated with different scan speeds, as schematically shown in Fig. 3(c). This was performed in air at $50 \% \mathrm{RH}$ and the DC bias voltage was 
kept at $-2.0 \mathrm{~V}$. The surface potentials of all the modified regions were increased compared with the surrounding areas. Figure 3(d) shows the cross-sectional plots obtained along the dotted line G-H in Fig. 3(b). The surface potential shift was increased as scan speed was decreased. Therefore, we can conclude that the number of oxidized 11-FUT molecules depends on scan speed.

To confirm that the surface potential of an 11-FUT SAM can be controlled with reversibility, a series of surface modifications was performed, as illustrated in Fig. 4(a). First, a $4 \times$ $4 \mu \mathrm{m}^{2}$ square region was scanned with a DC bias voltage of $-2.0 \mathrm{~V}$ to oxidize the 11-FUT molecules in the scanned area. Next, the oxidized 11-FUT molecules in the $2 \times 2 \mu \mathrm{m}^{2}$ square were reduced by scanning with a DC bias voltage of $0.0 \mathrm{~V}$. Then, the reduced molecules in the $1 \times 1 \mu \mathrm{m}^{2}$ square were oxidized again by scanning with a DC bias voltage of $-2.0 \mathrm{~V}$. Finally, the tip returned to the center of the scanned area with a DC bias voltage of $0.0 \mathrm{~V}$. This modification was performed in air at $50 \% \mathrm{RH}$ and the scan speed during the surface modification was kept at $5 \mu \mathrm{m} / \mathrm{s}$. Figures $4(\mathrm{~b})$ and $4(\mathrm{c})$ show the topographic image and the corresponding surface potential image, respectively, obtained after the modification. The topographic image showed a few protrusions. However, these protrusions did not correspond to the shape of the scanned areas and thus it is speculated that they were fabricated by an abrupt collision of the tip and the sample. On the other hand, the surface potential image clearly showed the same contrast as the 4th illustration in Fig. 4(a). The respective regions in the surface potential image correspond to the (1) unscanned, (2) oxidized, (3) reduced, (4) reoxidized, and (5) rereduced regions. Surface potential was reversibly changed as applied DC bias voltage was changed, which suggests that the redox states of 11-FUT molecules in the modified areas were repeatedly manipulated.

\section{Conclusions}

11-FUT SAMs and a HDT SAM were locally modified by applying DC bias voltages to the SAMs using AFM, and the surface potential distributions of the modified areas were investigated by KFM. 11-FUT SAMs showed a positive surface potential shift in negatively biased areas and a negative surface potential shift on positively biased areas. On the other hand, no positive surface potential shift was found on a HDT SAM. The surface potential shift in the 11-FUT SAMs was strongly affected by humidity and scan speed; no effect of oxygen concentration was found. In addition, we successfully demonstrated the surface-potential reversibility of an 11-FUT SAM. The oxidization of the ferrocenyl groups was suggested as the origin of the positive surface potential shift.

\section{Acknowledgment}

This work was supported by a Grant-in-Aid for Scientific Research B from the Ministry of Education, Culture, Sports, Science and Technology (MEXT) of Japan, by a Grant-in-Aid 
Jpn. J. Appl. Phys.

for the Global COE Program, "International Center for Integrated Research and Advanced Education in Materials Science," also from MEXT of Japan, and by a research grant from Mazda Foundation. 
Jpn. J. Appl. Phys.

Regular Paper

\section{References}

1) C. E. D. Chidsey, C. R. Bertozzi, T. M. Putvinski, and A. M. Mujsce: J. Am. Chem. Soc. $112(1990) 4301$.

2) K. Uosaki, Y. Sato, and H. Kita: Langmuir 7 (1991) 1510.

3) G. K. Rowe, and S. E. Creager: Langmuir 7 (1991) 2307.

4) G. K. Rowe, and S. E. Creager: Langmuir 10 (1994) 1186.

5) T. Auletta, F. C. J. M. Veggel, and D. N. Reinhoudt: Langmuir 18 (2002) 1288.

6) A.V Tivanski, and G. C. Walker: J. Am. Chem. Soc. 127 (2005) 7647.

7) J. He, and M. Lindsey: J. Am. Chem. Soc. 127 (2005) 11932.

8) S. Fujii, S. Kurokawa, K. Murase, K-H. Lee, A. Sakai, and H. Sugimura: Electrochim. Acta 52 (2007) 4436.

9) R. R. Gagné, C. A. Koval, and G. C. Lisensky: Inorg. Chem. 19 (1980) 2854.

10) N. Oyabu, O. Custance, I. Yi, Y. Sugawara, and S. Morita: Phys. Rev. Lett. 90 (2003) 176102.

11) R. D. Piner, J. Zhu, F. Xu, S. Hong, and C. A. Mirkin: Science 283 (1999) 661.

12) M. Yasutake, Y. Ejiri, and T. Hattori: Jpn. J. Appl. Phys. 32 (1993) L1021.

13) D. Wouters, and U. S. Schubert: Angew. Chem., Int. Ed. 43 (2004) 2480.

14) R. Maoz, S. R. Cohen, and J. Sagiv: Adv. Mater 11 (1999) 55.

15) N. Saito, N. Maeda, H. Sugimura, and O. Takai: Langmuir 20 (2004) 5182.

16) H. Sugimura, S-H. Lee, N. Saito, and O. Takai: J. Vac. Sci. Technol. B 22 (2004) L44.

17) G. Bar, S. Rubin, T. N. Taylor, B. I. Swanson, T. A. Zawodzinski Jr., J. T. Chow, and J. P. Ferraris: J. Vac. Sci. Technol. A 14 (1996) 1794.

18) S. D. Evans, and A. Ulman: Chem. Phys. Lett. 170 (1990) 462.

19) M. Yang, Z. Zheng, Y. Liu, and B. Zhang: J. Phys. Chem. B 110 (2006) 10365.

20) J. Han, K.-H. Lee, S. Fujii, H. Sano, Y.-J. Kim, K. Murase, T. Ichii, and H. Sugimura: Jpn. J. Appl. Phys., 46 (2007) 5621. 
Jpn. J. Appl. Phys.

Fig. 1 (a) Schematic for explaining redox of a ferrocenyl group. (b) Schematic of a ferrocenylthiol SAM on a Au surface.

Fig. 2 (a) Schematic of a surface modification of the SAMs. 9 square regions were scanned by applying DC bias voltages. (b)-(i) Topographic images $[(b),(d),(f),(h)]$ and the corresponding surface potential images [(c), (e), (g), (i)] of the SAMs after the surface modification. An 11FUT SAM modified in air at $50 \% \mathrm{RH}[(\mathrm{b}),(\mathrm{c})]$, a HDT SAM modified in air at $50 \% \mathrm{RH}[(\mathrm{d})$, (e)], an 11-FUT SAM modified in air at $10 \% \mathrm{RH}[(\mathrm{f}),(\mathrm{g})]$, and an 11-FUT SAM modified in $\mathrm{N}_{2}$ gas at $10 \% \mathrm{RH}[(\mathrm{h}),(\mathrm{i})]$. (j) Cross-sectional plots obtained along the broken lines A-B, C-D, and E-F.

Fig. 3 (a) Topographic image obtained after the surface modification with different scan speeds. (b) Surface potential image obtained simultaneously with (a). (c) Schematic for explaining the surface modification of the SAM with different scan speeds. (d) Cross-sectional plots obtained on the broken line G-H.

Fig. 4 (a) Schematic for explaining the reversible redox change. (b) Topographic image obtained after the reversible redox change. (c) Surface potential image obtained simultaneously with (b). 
(a)

$[00] \stackrel{\circ}{\leftrightarrows}[00]$

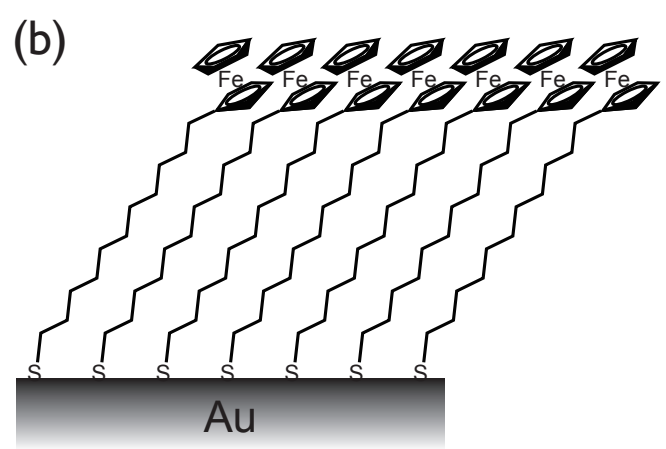

T. Ichii et al. Fig. I (200\%) 
(a)
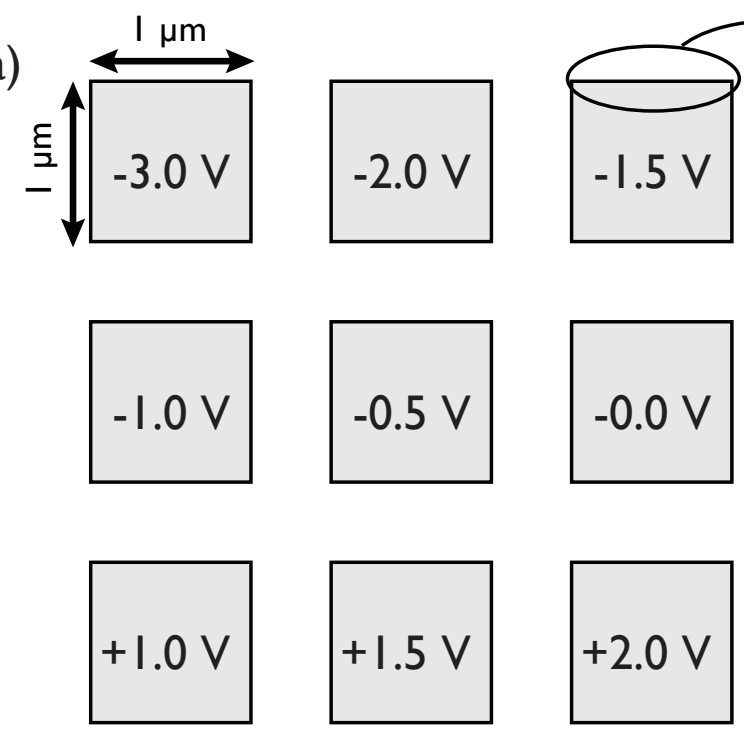

(b)

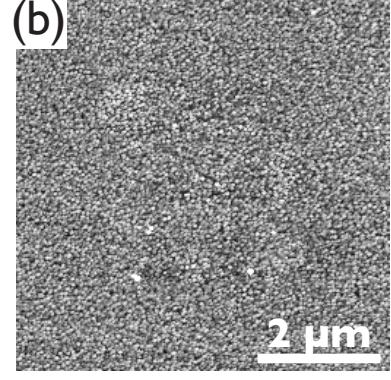

(c)
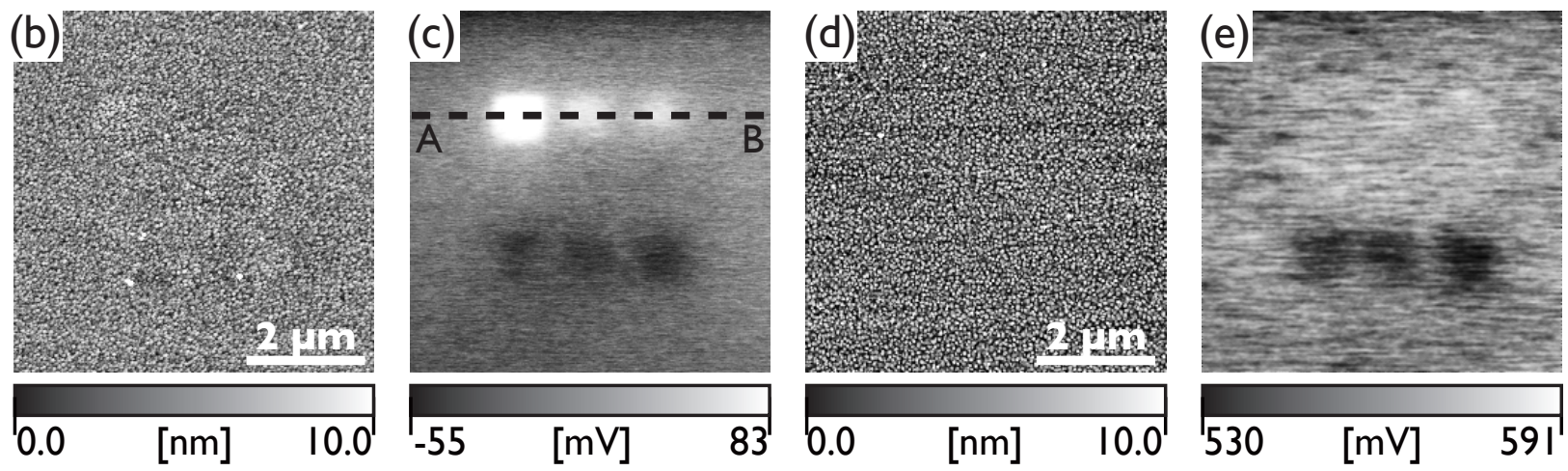

(f)
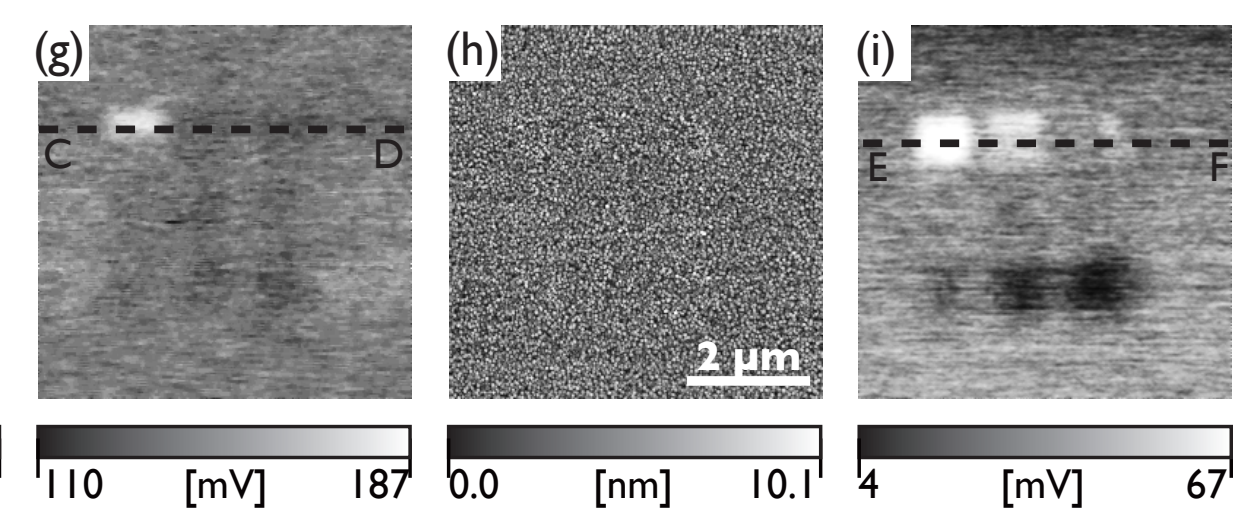

\begin{tabular}{lll}
\hline $0.0 \quad[\mathrm{~nm}]$ & 10.1
\end{tabular}
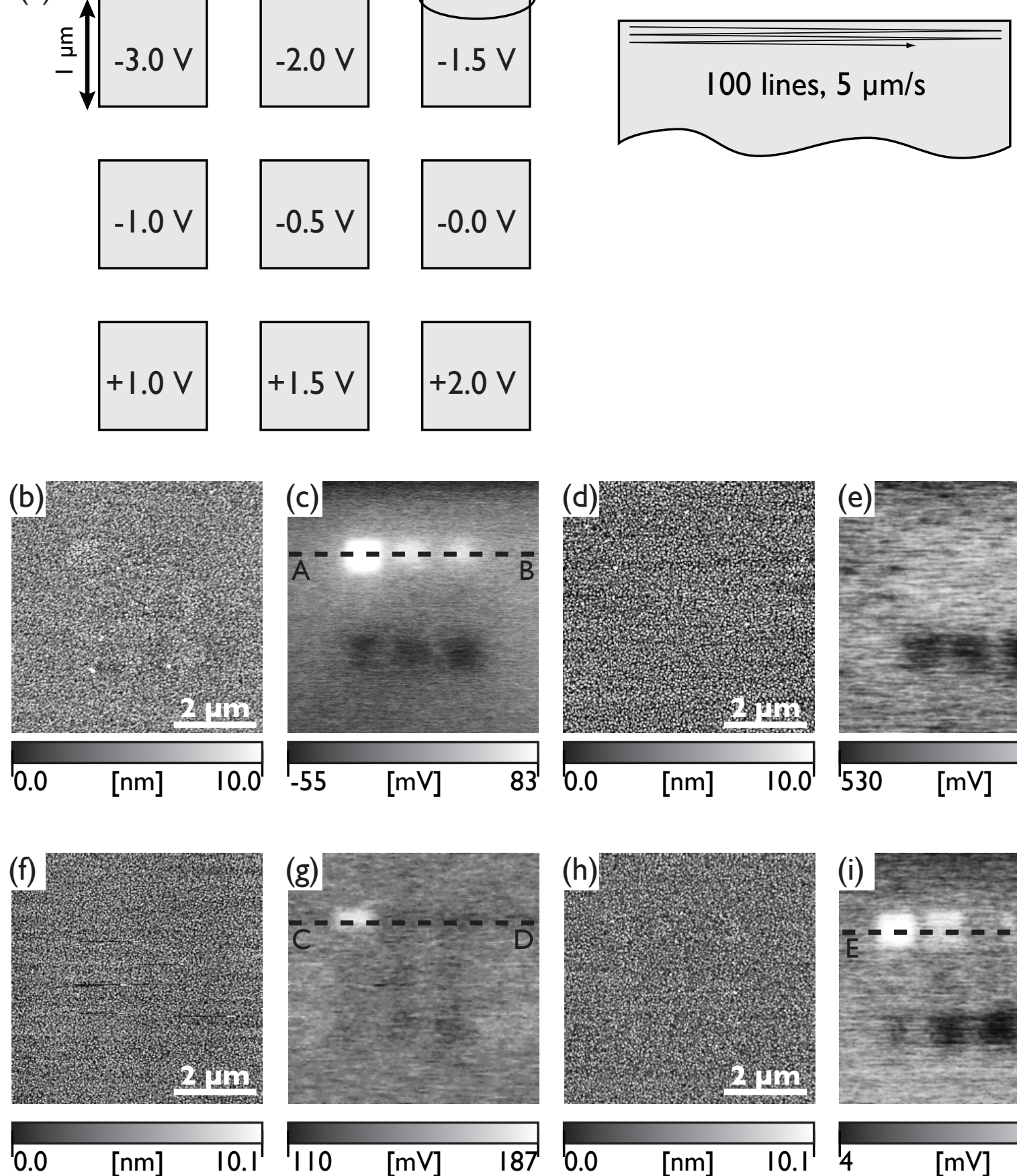

(j)

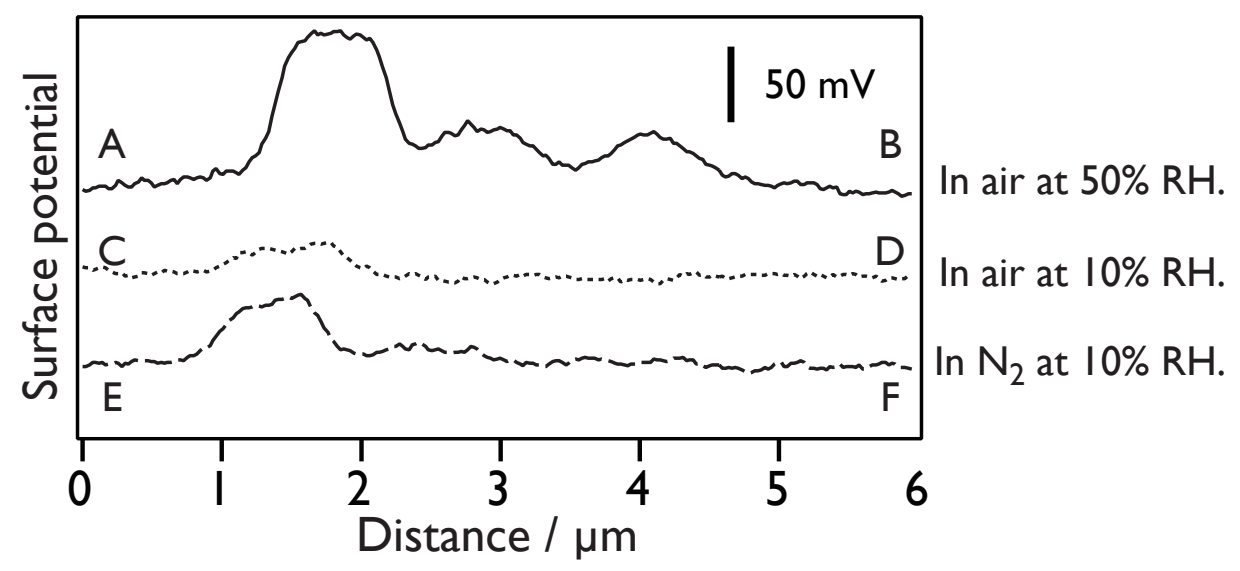

T. Ichii et al. Fig. 2(200\%) 
(a)

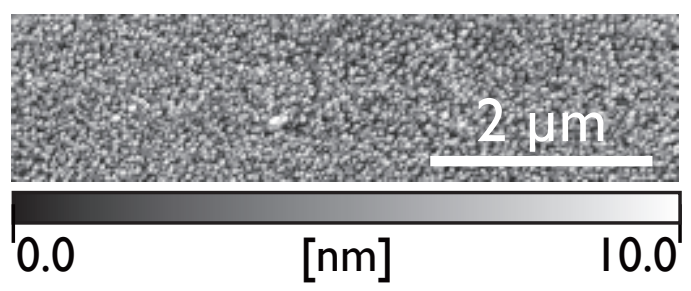

(c)

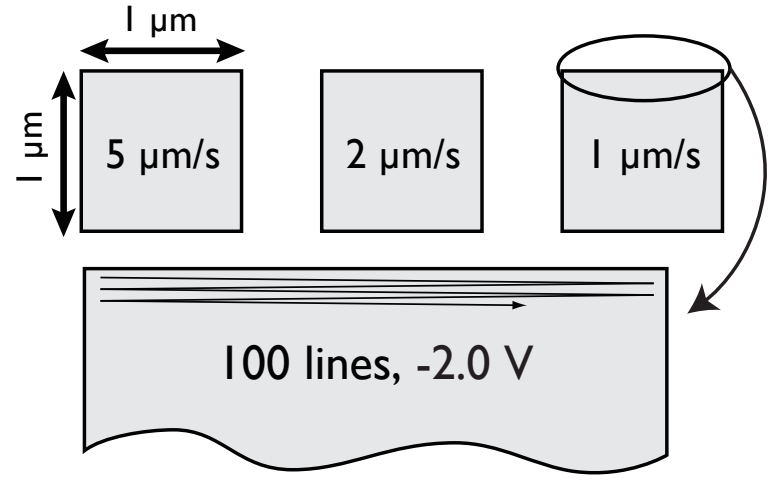

(b) $5 \quad 2 \quad$ । $[\mu \mathrm{m} / \mathrm{s}]$

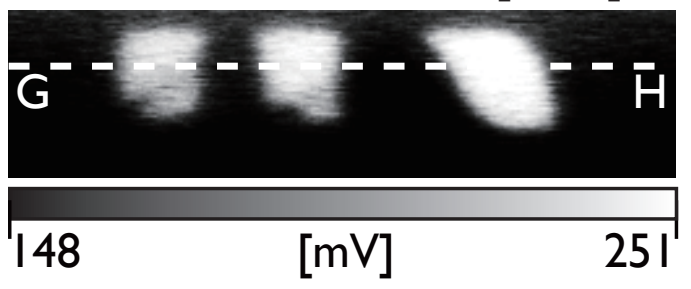

(d)

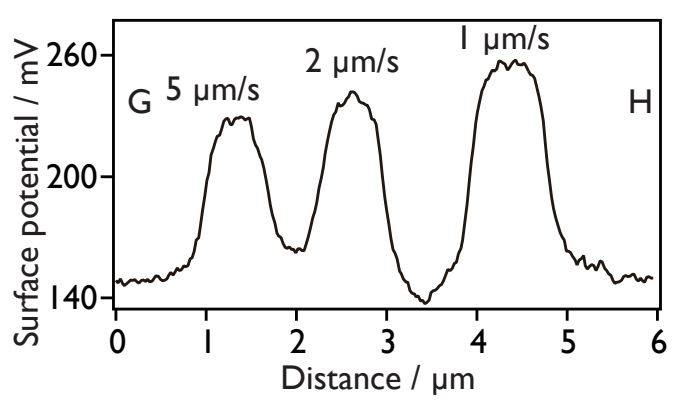

T. Ichii et al. Fig. 3(200\%) 
(a)
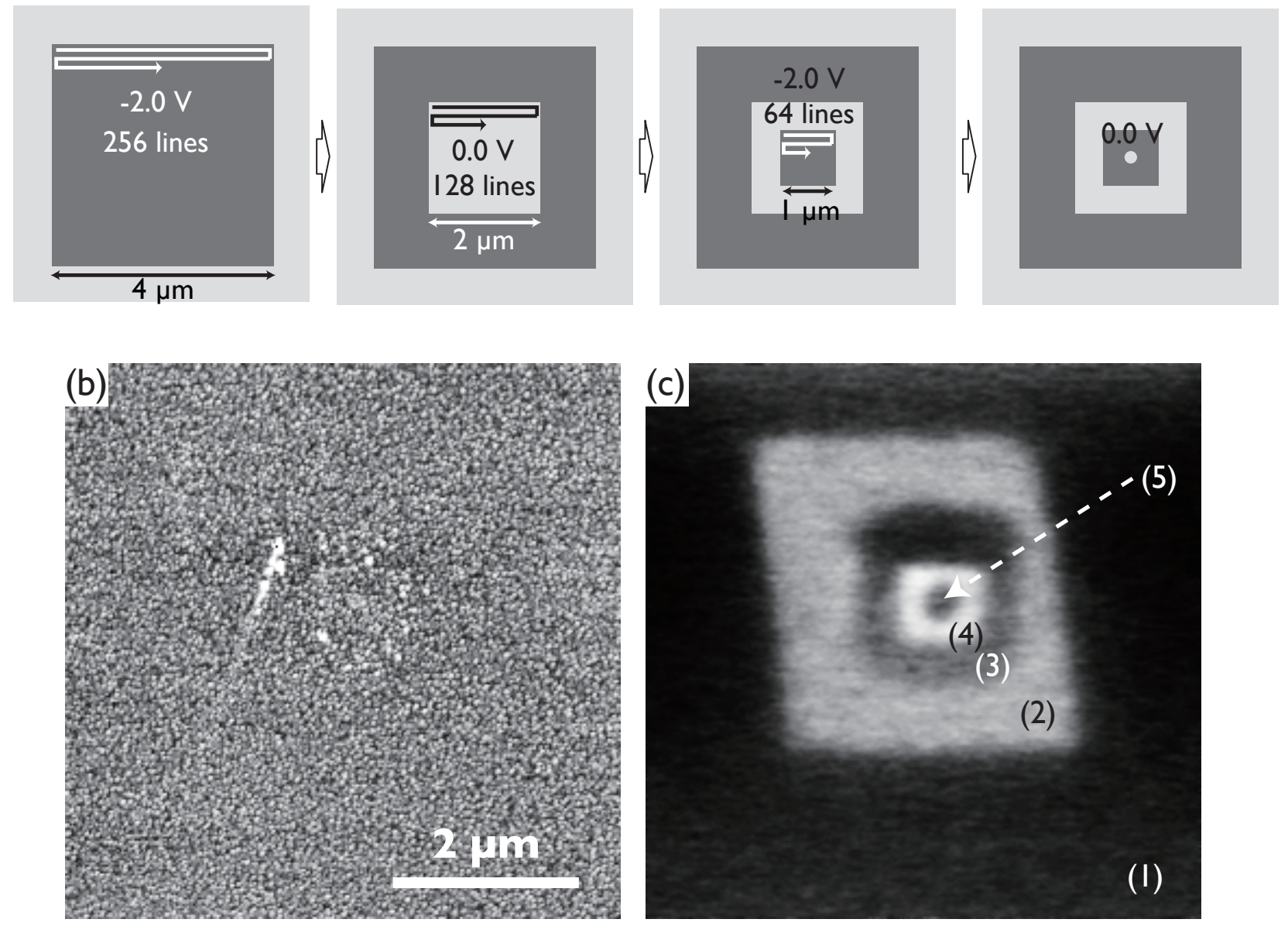

(c)

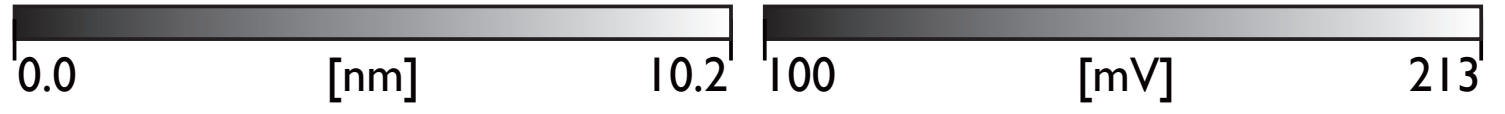

T. Ichii et al. Fig. 4(200\%) 